\title{
Micro-Economic Benefits of Peer-Producing Containerized Network Functions
}

\author{
Dewang Gedia1, Levi Perigo², Rahil Gandotra1 \\ ${ }^{1}$ Interdisciplinary Telecom Program, University of Colorado Boulder, Boulder, USA \\ ${ }^{2}$ Department of Computer Science, University of Colorado Boulder, Boulder, USA \\ Email: dewang.gedia@colorado.edu,levi.perigo@colorado.edu,rahil.gandotra@colorado.edu
}

How to cite this paper: Gedia, D., Perigo, L., \& Gandotra, R. (2020). Micro-Economic Benefits of Peer-Producing Containerized Network Functions. Open Journal of Business and Management, 8, 2285-2302. https://doi.org/10.4236/ojbm.2020.85140

Received: August 10, 2020

Accepted: September 21, 2020

Published: September 24, 2020

Copyright (c) 2020 by author(s) and Scientific Research Publishing Inc. This work is licensed under the Creative Commons Attribution International License (CC BY 4.0). http://creativecommons.org/licenses/by/4.0/ (c) (i) Open Access

\begin{abstract}
Discrete, non-virtualized network elements are characterized by large costs, limited functions, vendor lock-in, and limited orchestration. Virtualization technologies like virtual machines (VM) and containers have expanded the scope of virtual resource utilization through consolidation of workloads that were previously running on multiple servers by running them on a single server. With the advent of Network Functions Virtualization (NFV), industries are able to reduce the micro-economic factors associated with vendor proprietary model such as transaction costs and (physical and human) asset specificity to deal with vendor vulnerabilities in contractual relationships because Virtual Network Functions (VNFs) can virtualize dedicated networking functions that were traditionally performed by vendor appliances such as routers, switches, firewalls, and load balancers. Even though virtualization technologies (VMs and containers) and NFV have demonstrated their benefits in the market, little attention has been devoted to the development and adoption of containers to build VNFs. This research paper identifies micro-economic factors, such as transaction costs, associated with searching, buying, provisioning, and maintenance of vendor proprietary appliances and compares them with the coordination costs associated with the adoption of containerized VNFs. This comparative analysis could be used to identify the type of network operators that could serve as key organizers (the network operator who can benefit largely by adopting containerized VNFs) of an open source peer production model as well as other firms that could serve as individual contributors. Furthermore, to identify various rewards and incentives that a managerial firm can leverage to motivate its employees to participate in such an effort, a quantitative survey was conducted (with Tier 1, Tier 2 and Tier 3 Service Providers) to identify the managerial incentives such as bonuses, rewards, peer recognition, and promotion targeting varied network operator firms to accurately capture and analyze employee interests/motivation.
\end{abstract}


Finally, the paper shows a measuring framework to evaluate individual contributors based on the project modularity and indicates the viability of this model.

\section{Keywords}

Container, Interdisciplinary, Modularity, Motivations, Peer Production

\section{Introduction}

Network Functions Virtualization (NFV) promises lower operational costs for network service providers by offering automated elasticity, software and hardware independence, virtualized software infrastructure, service agility, and operating model changes (Valencia, Izzo, \& Polonsky, 2015; Gedia \& Perigo, 2018b, 2018c; Gandotra \& Perigo, 2018). With the advent of NFV, the industries are able to eliminate the microeconomic factors associated with vendor proprietaries such as transaction costs and asset (physical and human) specificity to deal with vendor vulnerabilities in contractual relationships. Virtual Network Functions (VNFs) can help virtualize dedicated networking functions that were earlier performed by vendor appliances such as routers, switches, firewalls, and load balancers. Even though the virtualization technologies (VMs and containers) have marked their presence in the current market, little attention has been devoted to the development and adoption of containers to build network functions (majority of the VNFs available today are still VM based). At "SDN NFV World Congress," Martin Taylor, CTO of Metaswitch raised this concern associated with the immaturity of the container ecosystem with regards to NFV deployment (SDN-NFV World Congress Agenda). This research paper presents a thorough analysis of microeconomic aspects that can help encourage service providers to transform their networking model from vendor proprietary based to open source software based adaptation. Figure 1 provides an overview of current vendor based proprietary model to open source VNF model.

Currently, service providers use the traditional proprietary model that uses vendor-specific network equipment as a part of their network infrastructure. As Figure 1 depicts, vendor devices have proprietary hardware and software (based on the networking application) that are managed by Network Operators. This proprietary model involves various contracts with vendors and service providers to sustain uninterrupted service delivery to the customers. With NFV, different application specific VNFs can be run on a single hypervisor thus diversifying the type of applications as well as optimizing resource utilization. This research paper shows various micro-economic costs associated with the current vendor proprietary method that can be overcome by using commons-oriented peer production mechanism (Bauwens, 2009). Commons-based peer production produces non-rival immaterial information goods that can be reproduced at 


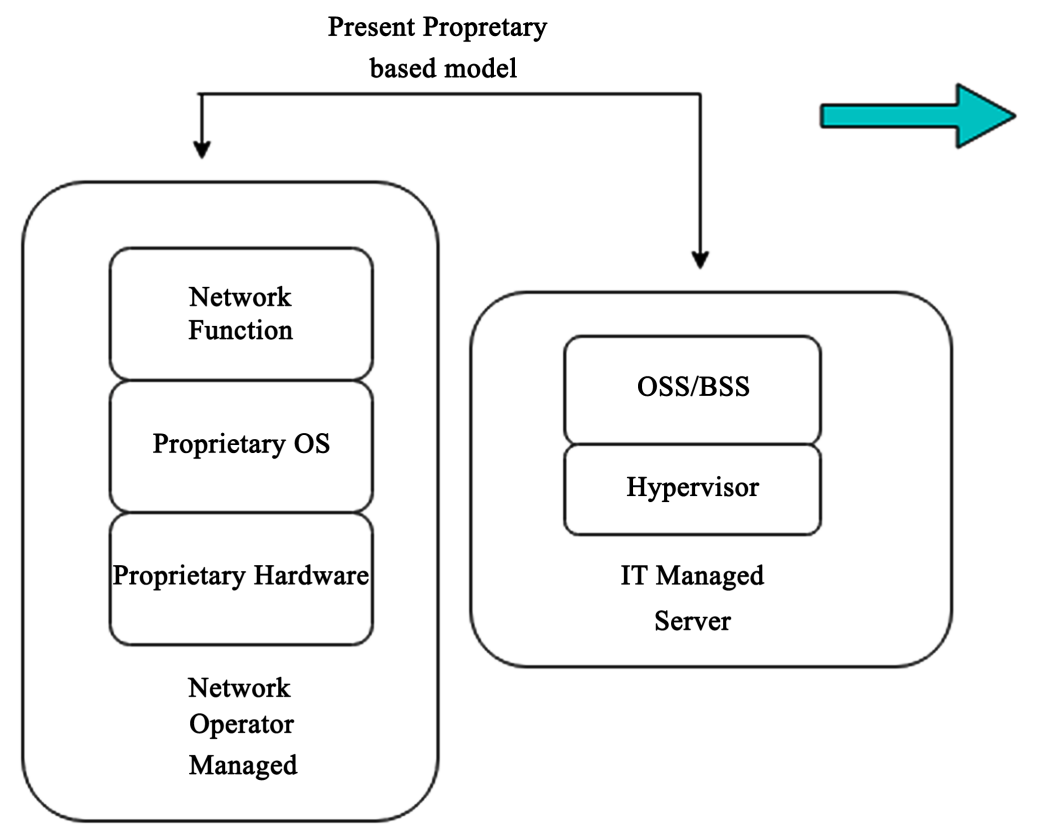

NFV based model

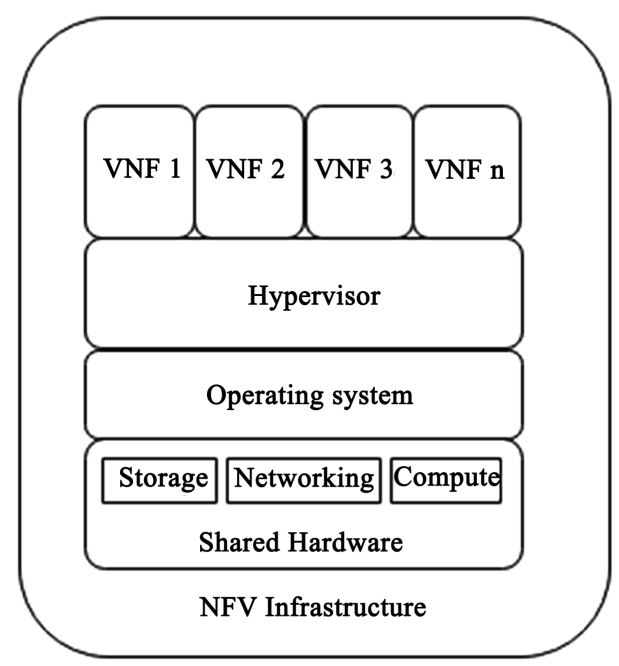

Figure 1. Vendor proprietary model versus NFV paradigm.

marginal cost as well as does not create tension between supply and demand (Bauwens, 2009; Benkler \& Nissenbaum, 2006). Given the economic advantages of Operations Support System (OSS) over the proprietary model, the research carried out in this paper states various viable conditions that can serve as a motivation for ISPs to participate in such a peer-production model. Additionally, the research shows which type of ISPs (Tier 1, Tier 2, Tier 3) may benefit by adopting peer-produced VNFs.

The remainder of the paper is organized as follows: Section 2 describes the primary research question and associated subproblems. Section 3 provides a literature review of related work. Section 4 demonstrates the methodology to answer the subproblems. Section 5details the results obtained for each subproblem. Section 6 concludes the paper and describes the future work.

\section{Research Question}

The research focuses on the question: "Can peer production model lower microeconomic costs for ISPs by encouraging open innovation to develop VNFs?" This research question is strategically divided into the following three subproblems that address an individual technological research aspect which would collectively answer the primary research question:

1) Is there sufficient mutuality of interests to get Internet Service Providers and individuals to commit to a peer production arrangement and thus, possibly take on the role of key organizer?

2) Can the process of developing VNFs and containers be made sufficiently modular to make peer production feasible? If so, what are the incentives that might motivate employee contributions? 
3) What are the favorable conditions for developing and adopting Containerized VNFs?

\section{Literature Review}

While there has been work related to the design of VM based VNFs, the industry lacks motivation for container based VNFs despite observing that container-based VNFs have proved advantageous over VM-based VNFs (Gedia \& Perigo, 2018a). In addition to the performance benefits, containers also prove advantageous economically since they provide better resource efficiency due to its kernel sharing properties compared to VM-based VNFs thus, offering multiple network functions on a single server. To further lower the CAPEX and OPEX by preventing vendor lock-in for an ISP, it is important to analyze micro-economic costs associated with containerized VNFs from the perspective of markets and firms. In "The Nature of the Firm," R. H. Coase attributed costs of coordination, or transaction costs (the costs associated with gathering of information), that are required to produce goods, negotiate (specially to prevent any risks of opportunism), determine design, and price and quantity of the good being produced serve as the primary reasons for the existence of firms (Coase, 1937). The authors of "Electronic Markets and Electronic Hierarchies" successfully validate their analytical framework through the primary research question proposed Would reducing the costs of coordination with the help of information technology, shift economic activity coordination toward more use of markets than hierarchies? (Malone, Yates, \& Benjamin, 1987). Their research also associated Coase's idea of telegraph as an example to show that it increased the number of economic actors involved in remote transactions in markets, thus favoring hierarchies. Contemporary changes in information technology paved the way for electronic communication and its associated electronic brokerage and electronic integration effects that lead to favor markets versus hierarchies (Besten, Dalle, \& Gallia, 2008). Thus, manifesting a product (example: travel tickets) that is highly asset specific and complex can be conveniently marketed to the customers that involve less hierarchical transactions. This clarifies the overall shift toward markets versus hierarchies by reducing costs of transaction due to advancement in information technology. To understand motivations for firms to develop containerized VNFs, this research paper analyzed multiple micro-economic factors such as contractual vulnerabilities, cost of containerization, and mutuality of interest.

The author Benkler in chapter 3 of "The Wealth of Networks" describe a newly emerged form of production known as "commons based peer production," besides markets and firms, that has led to the success of various projects such as Linux, Apache Web server, Perl, and Python (Benkler, 2003). The author categorized commons into four types based on two parameters by providing examples, first, that is open to anyone or specific group and second, whether the commons system is regulated or unregulated. The author defines 
"peer production" as the one that produces goods in which individual action is self-selected and decentralized. The author then elaborates on the open source license, GNU License (that led to innovation of free software) as well as the terms "copyright" and "copyleft." (Benkler, 2002) has identified the success of peer production in academic research and knowledge, NASA Clickworkers, Wikipedia, Slashdot, Project Guttenberg, Little League, Bowling Leagues, and Book clubs in his research "Coase's Penguin, or, Linux and The Nature of the Firm." He proposed three pre-conditions for the success of peer production: modularity of projects, modules should be sufficiently fine-grained for participants to accurately match their skill set, low-cost integration of contribution by participants (that can be achieved through modern IT). Another factor that enhances the peer production model is "accreditation and relevance" (Benkler, 2002). In "Economics of social production," the author highlighted the motivation and feasibility conditions of peer production (Benkler, 2003). Josh Lerner and Jean Tirole state "Modularity" as a favorable characteristic in maximizing the participation from individuals based on their interests (Lerner, \& Tirole, 2002). They define the term "Modularity" as an overall project that is divided into smaller well defined tasks (modules) that the participants can tackle individually. Referencing (Lerner, \& Tirole, 2002), this research paper divides VNF components into multiple modules that can collectively encourage participation in peer production of containerized VNFs to address the problem associated with development of open-source containerized VNFs (Laghrissi \& Taleb, 2018).

(Osterloh, Rota, \& Kuster) attributed the contribution of individuals to open source projects based on two motivations:

Intrinsic Motivations: This is sub-classified by (Lindenberg, 2001) into two sub-kinds of motives: Enjoyment-based Intrinsic motivations and Obligation-based Intrinsic motivations. Enjoyment-based Intrinsic motivations were attributed by Deci and his group (Deci, \& Koestner, 1999) as the one that is done for mere pleasure like reading a novel or playing football. Obligation-based Intrinsic motivations as termed by (Frey, 1997) are the one based on tax morale or environmental ethics.

Extrinsic Motivations: This second category of motivations relies on satisfying needs indirectly through monetary compensation such as job promotion/opportunities, rewards, bonuses, and peer reputation/recognition.

(Osterloh, Rota, \& Kuster) identifies five different types of intrinsic and extrinsic contributors: commercial service providers, lead users, reputation investors, fun seekers, and members of the tribe. Incentives for "commercial service providers" include adopting a business model that can make money by offering support services to open source software users (Red Hat provides software support or IBM provides compatible hardware). "Lead users" can gain non-monetary benefits by tailoring the software to their own needs by contributing to the open source that can also be amended by other software users for enhancing the quality. "Reputational investors" are those who consider open 
source as a platform for making money indirectly by signaling their capabilities. However, empirical evidence (Kogut \& Metiu, 2001) shows 82\% of active Apache contributors made only one or two contributions. "Fun seekers" as intrinsic motivators contribute solely because they experience strong satisfaction from creating "something that works". Finally, "Members of the tribe," are intrinsically motivated by the gift-giving culture and contribute only because they like "helping others".

To understand these motivations for peer participation in containerized VNF development, this research paper conducted a survey with a small group of ISP employees to learn their motivations to be involved in such an effort. The survey responses validated Osterloh's (Osterloh, Rota, \& Kuster) peer production motivations thus, aiding ISPs to help determine the viability of such an effort.

To address the problems indicated in the review of literature, this research paper will make a novel contribution to the existing body of literature that prior implementations lacked by performing micro-economic analysis for peer-production of containerized VNFs as a viable alternative to vendor-based proprietary model for ISPs to understand cost savings and maximize efficiency from their networks.

\section{Research Methodology}

This research paper evaluates the benefits of peer-producing open source SDN-VNFs compared to a proprietary based vendor lock-in approach. In "The Federal Communications Commission," Coase attributed innovation to intellectual property rights model (Coase, 1959). Another economist Garrett Hardin argued that property rights (privatization) or a management system are needed to eliminate the "Tragedy of the Commons" that is associated with overuse or undersupply of shared resources (Hardin, 1968). However, in the newer model of peer-production, the chance of overuse is slim as intellectual resources are abundant (software) that can be reused at lower marginal costs. This research paper compared various contractual vulnerabilities, as posited by (Ang \& Beath, 1993) that are faced by service providers using a vendor proprietary model with the vulnerabilities that are present in peer-produced VNFs to identify whether peer production provides any advantages over the former.

To understand whether there is sufficient mutuality of interest for ISPs to engage in peer production, the research conducted in this paper studied the survey results obtained by multiple survey organizations such as Ovum and International Data Corporation (IDC). This research paper also describes various VNF modules that may assist the development of peer-produced VNFs per ETSI NFV design considerations. To create a well-defined peer production arrangement, the need of willing talent needs to be in place for it. Given the advantages of container-based VNF adoption, there seems to be various incentives for service providers and vendors to participate in such an arrangement. To understand this, this research paper analyzed various Intrinsic and Extrinsic motivations as 
elaborated by (Osterloh, Rota, \& Kuster), and highlighted the responses of the quantitative survey to the ISP DC employees about peer-production participation.

In the first subproblem, to understand whether there is sufficient mutuality of interest, this research analyzes various transaction costs (as well as other microeconomic factors) associated with searching, buying, provisioning and maintenance of vendor proprietary appliances. These costs would then be compared with the coordination costs associated with adoption of containerized VNFs. This comparative analysis of the data would then be used to identify the network operators that can serve as key organizers (the network operator who can benefit largely by adopting containerized VNFs) of peer production model and the other firms (who benefit marginally) would serve as individual contributors. Further, in the second subproblem the research analyzes various rewards and incentives (in the form of internal IT investments) that a managerial firm can leverage to motivate its employees to participate in such an effort. A quantitative survey was carried to help identify the managerial incentives such as bonuses, rewards, peer recognition, and promotion targeting varied network operator firms to accurately capture and analyze employee interests/motivations. Lastly, in third subproblem, the research creates a measuring framework to evaluate individual contributors based on the project modularity. The unique modular framework constructed in this research identifies peers working on a similar objective/task whose objectives can be evaluated by the expertise (based on working profile) of other peers.

\section{Results and Analysis}

The section describes the micro-economic analyses done to understand the micro-economic factors associated with peer-production of container-based VNFs to answer the primary research question.

\subsection{Is There Sufficient Mutuality of Interests to Get Internet Service Providers and Individuals to Commit to a Peer Production Arrangement and Thus, Possibly Take on the Role of Key Organizer?}

To identify whether a peer-production approach to build containerized VNFs would offer any advantages over proprietary-based vendor approach, the research compares and analyzes multiple micro-economic contractual vulnerabilities (specificity, sunkness, uncertainties regarding ex-ante cost estimation, client being held up, opportunity, and other costs) associated with VNFs (software products). The following differences were found in this research after comparing various contractual vulnerabilities that are faced by service providers using a vendor proprietary model with the vulnerabilities that are present in peer-produced VNFs.

1) Specificity (time/asset) and Sunkness (costs/learning): While the current 
vendor proprietary approach involves various time and asset (buying, operating, and maintaining costs associated with physical networking devices) specificity depending on contractual relationship with the client (Service Provider) and vendor (Network Equipment Manufactures (NEM)), peer production also involves similar asset specificity due to various intellectual and hardware resources that are put into building infrastructure (Osterloh, Rota, \& Kuster). In a vendor proprietary model, every vendor device, before being put into production, involves vendor-specific training to configure and manage. However, in the case of peer-produced VNFs, the service providers that are a part of peer production can save these sunk costs as well as reduce vendor lock-in. Thus, peer-produced VNFs have smaller sunk costs than those associated with the vendor proprietary model.

2) Uncertainties regarding ex-ante cost estimation: It has been proved in the prior economic literature (Ang \& Beath, 1993; Osterloh, Rota, \& Kuster) that contractual vulnerabilities pose difficulty in estimating ex-ante cost (costs that are estimated before the beginning of contract). However, in peer production, the participants are motivated by reciprocal altruism that can well define the task (Susan \& Glenn, 2014). Likewise, efficient governance mechanism in peer production of VNFs may serve as an incentive for service providers to achieve the goals that are required in an ISP infrastructure by becoming a key organizer (by participating as a leader of the peer production arrangement of SDN-VNF) in an early stage (Li \& Chen, 2015).

3) The client being held up: The current vendor proprietary model requires a service provider to be highly dependent on the need to install, configure, manage, and support vendor devices that is constrained by the vendors' expertise. However, the peer production approach would reduce these contractual vulnerabilities as the employees of service providers (participants of peer production) would have a better knowledge of the methods to deploy, manage, and support VNFs.

4) Opportunity costs: Contractual relationships between an ISP and a vendor pose opportunity costs (value lost due to non-reusability of good) for vendors since the custom code developed for an ISP cannot be reused for a different ISP. However, with peer production, participants can tailor the custom code needed for their respective ISP without bearing contractual opportunity costs.

5) Other costs: The vendor proprietary approach poses additional marginal costs of reproduction as the software used on vendor networking devices are license specific. However, peer-produced VNFs can reduce costs associated with reproduction and distribution with GPL public licenses. Moreover, informal and extralegal dispute resolution in the case of contract violation creates additional costs for vendors and ISPs.

Given the benefits that peer production has over contracts, it is beneficial for a service provider to adopt a peer production approach to create VNF applications that reduce the vulnerabilities associated with the contracts. 


\section{Is there sufficient Mutuality of Interests?}

In (John, et al., 2017) proved that NFV promises reduced IT/network operations expenditure by bringing automation to provide centralized control of the network behavior and zero-touch provisioning of the network equipment. (Dedrick \& West, 2003) demonstrated that the cost (of hardware and software) served as major motivations for open source adoption.

The research conducted by Ovum (Walker, 2012) suggested that costs associated with IT/network operations in a network operator/service provider account for $18 \%$ of the total revenue. Of this, $60 \%$ (US $\$ 126$ bn) is spent on providing salaries to the IT/operations staff. (Walker, 2012) suggested to decrease operating risks and cost base of service providers, additional service projects can be created by the transfer of employees to NEMs. The research (Walker, 2012) suggested that containerized VNFs can significantly reduce operating costs for service providers.

The OPEX exceeds CAPEX per the research conducted by John in (John, et al., 2017) is as shown in Figure 2.

Similar research was conducted by International Data Corporation - premier global provider for market intelligence, advisory services, and events for information technology, telecommunications and consumer technology markets reported that due to the global economic instability, intense competitive pressures, and pressures to decrease CAPEX spending has led service providers to encourage vendors to streamline their products by adopting virtualized software products rather than offering non-agile hardware devices (Hawkins, 2017). The findings also revealed that NEMs collectively generated $\$ 124$ bn worldwide by selling their infrastructure devices. Vendors that were part of the study included Cisco, Juniper, Ciena, Ericsson, Huawei, NSN, ZTE, and Alcatel-Lucent.

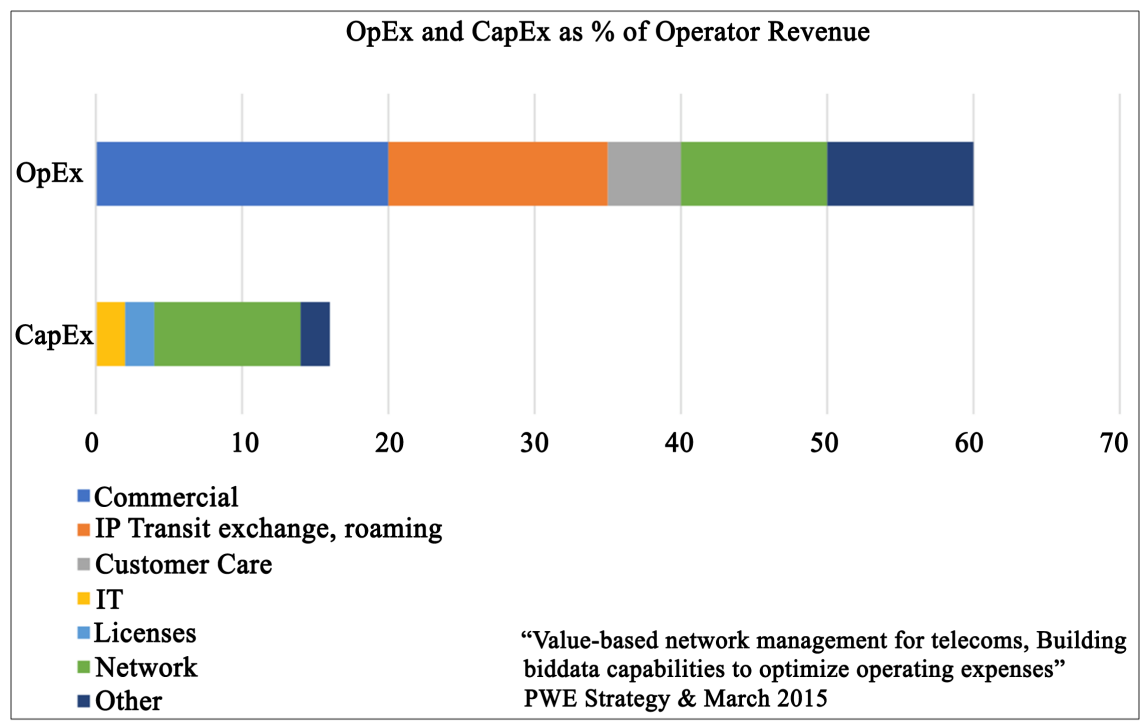

Figure 2. Cost breakdown for telecom operators. Source: "automating service creation and provisioning for creative Ethernet services". 
Per the data, network operators/service providers (users of networking devices) can benefit economically by lowering OPEX and CAPEX if they adopt peer-produced VNFs (and not contract for them due to the vulnerabilities stated in this subproblem) rather than buying/contracting networking devices.

In Coase's Penguin (Benkler, 2002), Benkler indicated that the sustainability of any open source project depends on the number of contributing individuals. He suggested that a project requiring thousands of person hours could be drawn on the expertise of fifteen or thirty thousand individuals in an open source project thus, lowering the personal cost of participation by diverse motivations. In "Some Simple Economics of Open Source," authors (Lerner, \& Tirole, 2002) indicated incentives for firms to participate in open source movement. One of the incentives for firms to participate in such a movement is that visibility (of key employees) helps attract more talented individuals thus, providing a strong incentive to existing employees. Another commercial advantage for NEMs (vendors) participating in an open source movement is that they can adopt a "reactive" strategy. As indicated by Lerner and Tirole, such participation by commercial firms into open source projects can help them build complementary products/services or sell "support" to open source users. Thus, vendors of networking devices have a significant incentive to provide a commitment to such an open source movement and create a business model by offering complementary services and support to VNF users. An additional strategy that (Lerner, \& Tirole, 2002) indicate is to take a proactive role in code development process by releasing their proprietary code. This is an especially useful strategy for smaller vendors (that are running out of business) of networking devices to make profits in the complementary segment (by offering additional proprietary services that are not provided in open source) of open source VNFs. Similar results were indicated by (Athey \& Ellison, 2014) who identified that the firms could receive substantial long-run benefit by bearing short-term costs of providing programmers. This benefit can be realized by providing support to the users (service providers) of VNFs. For the above mentioned contractual vulnerabilities and the presence of mutuality of interests among ISPs (users) and vendors, peer producing VNFs can serve as an economically viable model compared to contracting for networking equipment.

\subsection{Can the Process of Developing VNFs and Containers Be Made Sufficiently Modular to Make Peer Production Feasible? If So, What Are the Incentives That Might Motivate Employee Contributions?}

To realize pre-conditions (Benkler, 2002) for peer production of VNFs, this research referred to the ETSI-NFV framework to identify distinct modules for encouraging peer participation. ETSI has defined NFVI benchmarking aspects for it to meet the standards set by legacy hardware successfully but, at a lower cost. To tailor modules, NFVI architecture framework offers numerous reference 
points that need to be considered to develop a successful VNF. These reference points can be categorized into different modules for establishing a successful peer-production arrangement. The modules need to be produced for realizing carrier-grade deployment of containerized VNF:

1) Virtualization Layering and NFVI Support:

Virtualization serves as the most important layer of the architecture. This layer abstracts the hardware resources and provides anchoring to VNFs. Hypervisors serve as a tool for realizing the virtualization layer. The objective of this module would be to test the different hypervisors available that are most suited for VNF container deployment by ensuring independence between hardware resources, the virtualization layer, and diverse VNFs. Besides independence, other performances and better virtualization design need to be investigated for it to support different VNFs.

2) VNF Software Architecture:

NFV requires distinct network functions such as routing, switching, firewall, load-balancer, and NATing to be converted into software packages known as VNFs. These software packages of network functions can be achieved using the technique of decomposing whereby software developers create small modules of a VNF based on scalability, reusability, and efficiency. Thus, ensuring fine-grained contribution that can collectively form a complete VNF function by integrating different software modules.

3) NFV Management and Orchestration:

Challenges such as end-to-end network mapping, instantiating appropriate VNFs at the intended location, and portability to sustain the creation of new VNFs are presented due to the decoupling of VNF from underlying hardware. This module deals with the creation of management and orchestration interface for accurate identification of faults that can help recovery of the network in the event of failure. Further, the module requires the expertise of software developers to write the code, and network engineers to design network architecture which involves defining locations of NFV placement. Software developers need to work with existing OSS/BSS, and hardware resource management systems.

4) Performance:

Since VNFs are assigned less resource than those available on the physical hardware, performance may degrade in the event of higher workloads. Therefore, new mechanisms need to be developed that can split the workload into two VNFs and maintain the performance metrics. It is necessary for the developer to minimize performance degradation while allowing VNF container portability on NFVI.

5) Reliability:

To provide similar reliability offered by legacy vendor devices such as higher cost efficiency, high availability, no single point of failure, fault detection, and recovery mechanisms, this module would require the expertise of Quality Assurance Engineers to evaluate and test VNF module metrics. 
6) Security:

The use of hypervisors may expose vulnerabilities that may be exploited to create an attack vector and compromise VNFs. Network security engineers are required to ensure secure boot mechanisms are employed in hypervisors that use appropriate calls. Shared storage and shared networking expose a different set of vulnerabilities that need to be studied to create a secured NFVI.

To create a well-defined peer production arrangement, there needs to be a place for willing talent. Given the advantages of Container-based VNF adoption (Gedia \& Perigo, 2018a), there are incentives for service providers and vendors to participate in such an arrangement.

Referencing Osterloh's research about Intrinsic and Extrinsic open-source participation motivations, a quantitative survey was conducted in this research on "Motivations for Peer-Production Participation" to obtain opinions from ISP data center (DC) employees about contributing to the open source community. Results showed (Figure 4) the top selections were "Job promotion," followed by "Peer recognition/reputation." The participants of the survey included employees of network operator firms such as Charter Communications and CenturyLink, the wireless network operator T-Mobile, NEMs such as Cisco Systems and Palo Alto Networks, the cloud operator Amazon Corporate LLC, and content provider Facebook. The survey received eight out of fifty responses from the participants as shown in Figure 3.

Figure 4 indicates the motivations stated by the survey participants to engage in a peer production arrangement. Some attributed their motives to fulfill the "gift giving" experience while others attributed it to "career development." The responses bolstered intrinsic and extrinsic motivations as argued by Osterloh (Figure 5).

As the survey responses indicate, extrinsic motivations surpass intrinsic motivations, providing an indication to firm managers that extrinsic motivations such as job promotion and peer recognition can encourage greater employee participation in peer production of VNFs. Further, modularization and strong extrinsic motivations were found to encourage peer participation in open source peer production of VNFs.

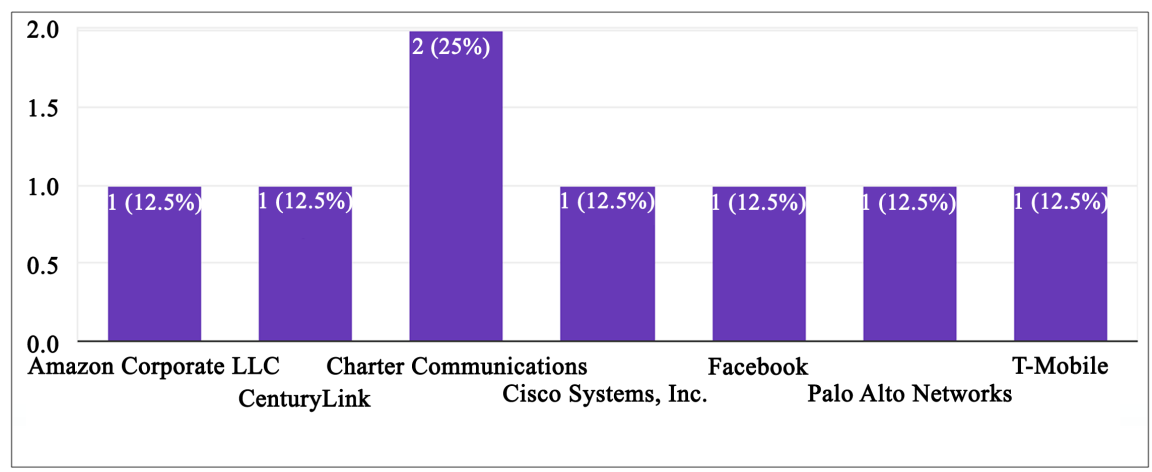

Figure 3. Participants of survey from ISPs. 


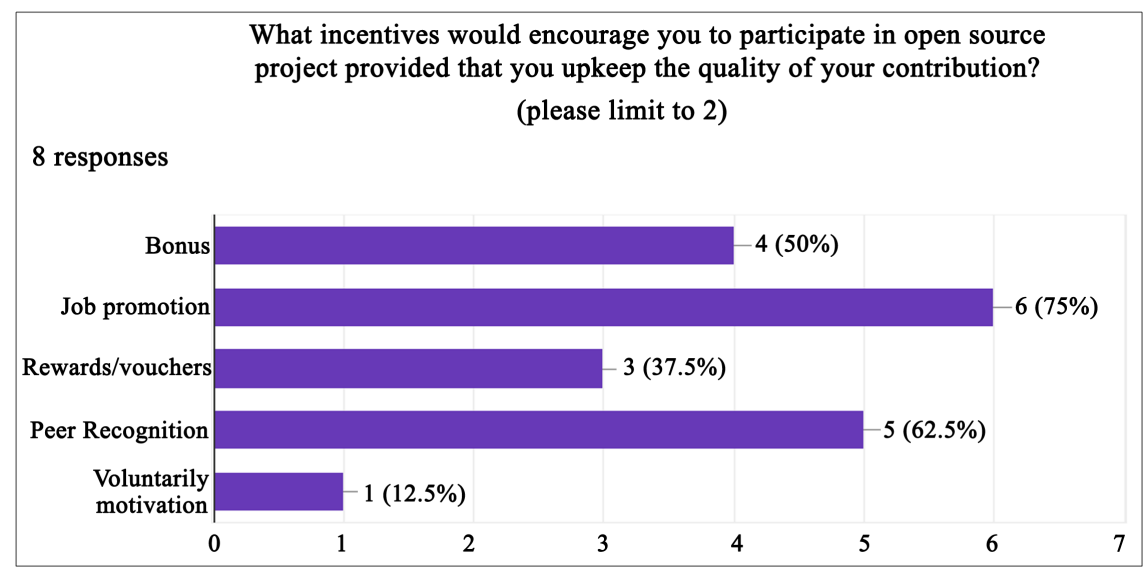

Figure 4. Incentives for employee open source participation.

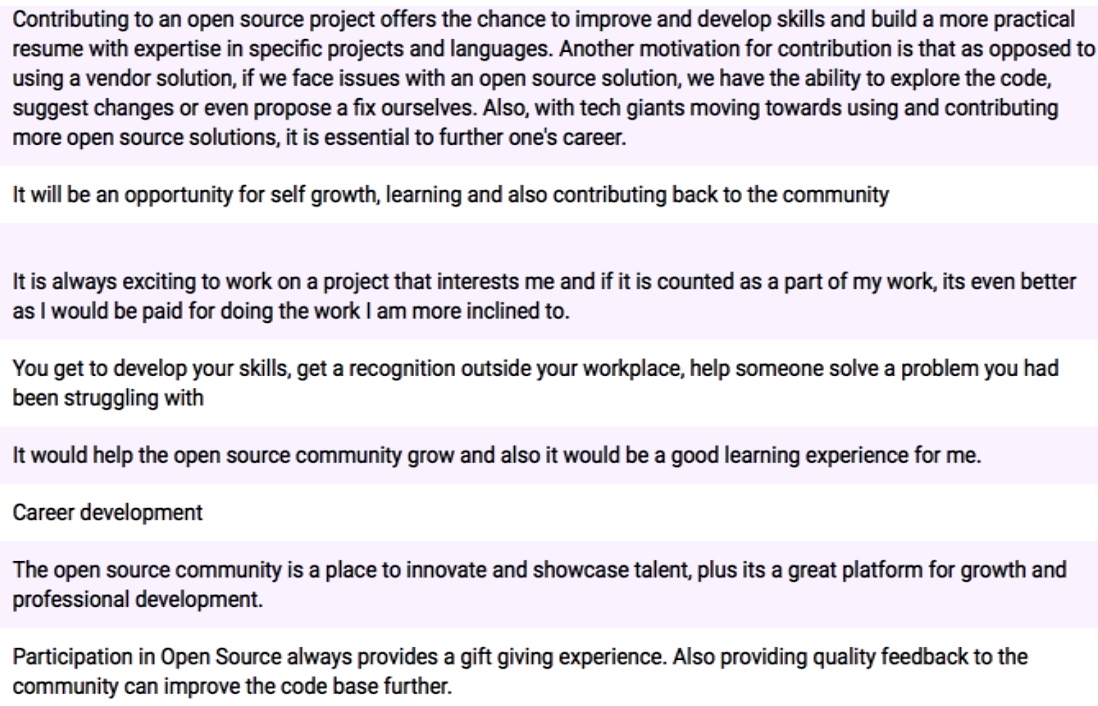

Participation in Open Source always provides a gift giving experience. Also providing quality feedback to the community can improve the code base further.

Figure 5. Motivations for open source participation.

\subsection{What Are the Favorable Conditions for Developing and Adopting Containerized VNFs?}

(Osterloh, Rota, \& Kuster) explained three interlinked characteristics that ensure efficient concurrence in design and testing of peer-produced product. They are listed as follows:

1) Open source software's source code should be produced under the public license that ensures public ownership by allowing the reader to have read access to the source code, make, and distribute copies, modify the program and distribute the program under the same license terms. One such license is that of "Copyleft" provided by General Public License (GPL).

2) User-driven distributed knowledge production in the form of rapid feedback cycles provides a unique model of production that enables concurrence in design and testing of various modules. In contrast to traditional software production models by firms, open source production receives more feedback (by 
users who deploy the software) that helps detect software issues by "debugging."

3) Voluntary "virtual communities of practice" are characterized by the dramatic reduction of search costs to match willing talents to the tasks. Individuals self-select the tasks based on their skillsets best matched. This not only prevents information losses (that are present in market and hierarchies), but also bolsters intrinsic motivation.

While the body of literature lacks significant effort for developing containerized VNFs that indicate lower transaction and operating costs (Laghrissi \& Taleb, 2018), Azhar Sayeed, chief architect at Red Hat, raised a scalability concern associated with containers for NFV. A service provider planning to deploy 1000 container-based virtual customer premise equipment (vCPEs) on a single server was only able to deploy 100 container vCPEs on a single server. This raises a concern - what type of service providers (users) are better suited to use containers for NFV? To address this concern, the research answers this subproblem by drawing a capacity cost curve that identified decreasing average costs as the number of container utilization increased.

For analysis, consider the cost of a server to be $\$ 1000$, and consider the marginal cost to be the cost associated with the learning curve required to deploy containerized VNFs. Now assume the capacity of a single server to be 100 containerized VNFs. Since VNFs are peer-produced (GPL license based), consider it to be available for free of cost. As shown in Figure 6, a service provider " $\mathrm{X}$ " starts deploying containerized VNFs gradually. If service provider " $\mathrm{X}$ " is a Tier III ISP that requires 100 VNFs. In that case, the average cost/container would exponentially decay from $\$ 1000$ (Point A in Figure 7) to $\$ 10$. Consider, a service

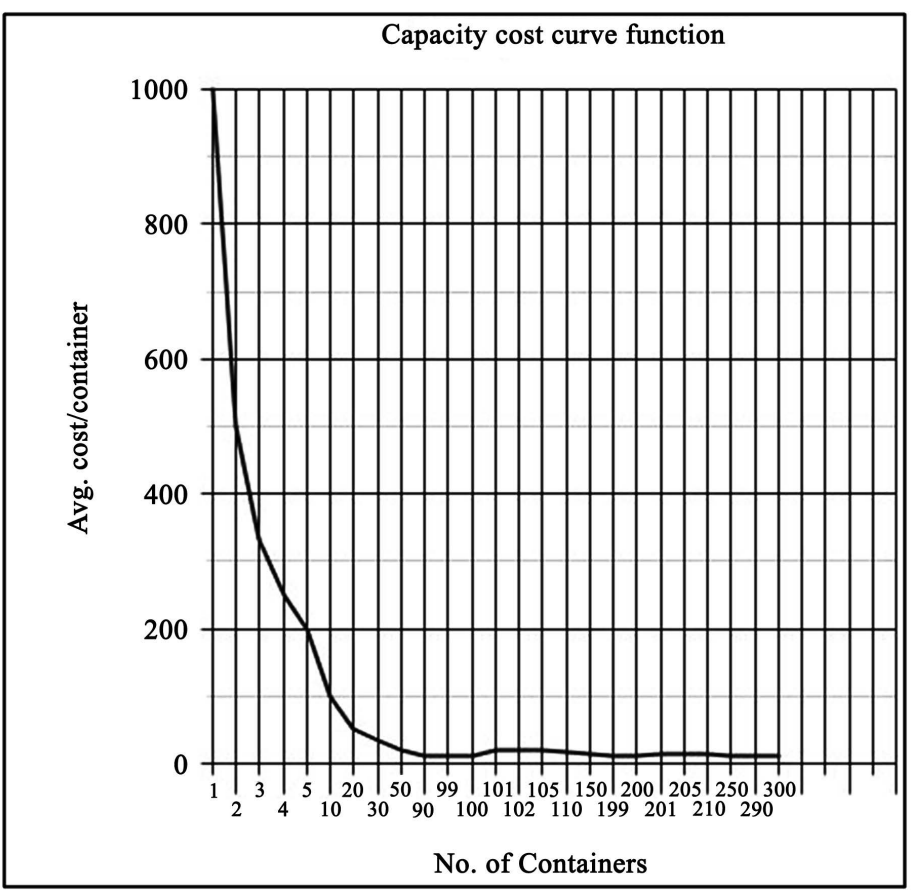

Figure 6. Capacity cost curve function 1. 


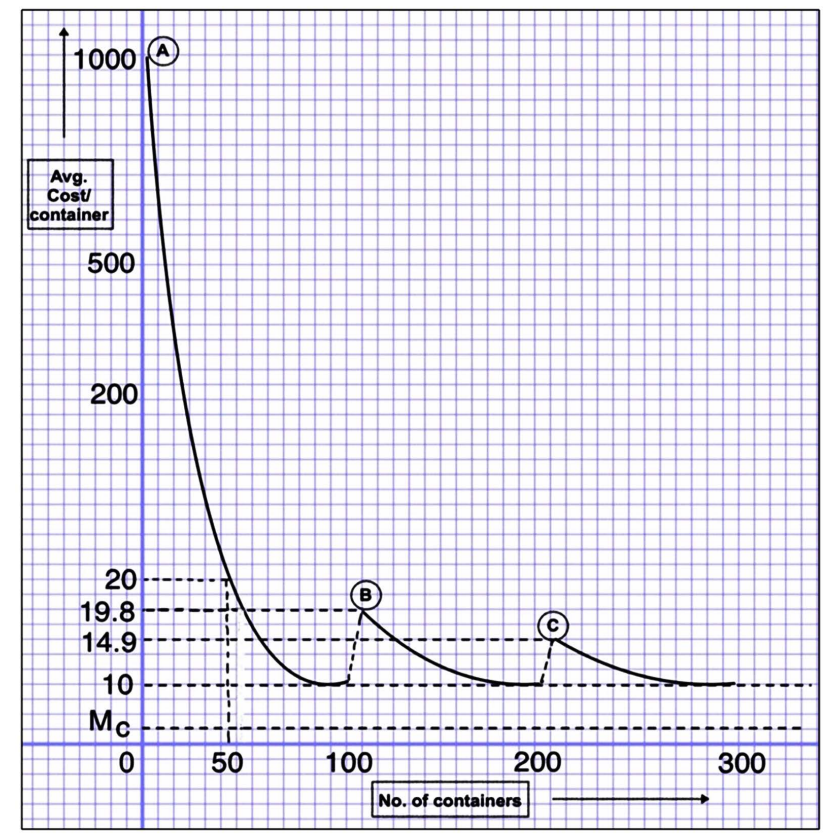

Figure 7. Cost curve function 2.

provider "Y" that is a Tier II ISP, that requires 200 VNFs. In this case, the capacity curve rises from $\$ 10$ (i.e., after server one is completely utilized and server two is purchased and deployed) to $\$ 19.8$ when an additional deployment of container on server 2 takes place and decays back to the $\$ 10$ mark. Consider, service provider "Z," a Tier I ISP that requires 300 VNFs. In this case, the capacity curve rises from $\$ 10$ (i.e., after server two is completely utilized and server three is purchased and deployed) to $\$ 14.9$ when an additional deployment of container on server 3 occurs and then again decays back to $\$ 10$. These discontinuities eventually converge to a constant straight line in the case of a large Tier I ISP like ISP "Z" depicting lower average cost/marginal container. This behavior illustrates the presence of economies of scale. These economies of scale will be present in larger ISPs which concludes that container utilization of VNFs are better suited to Tier I and Tier II service providers. Figure 7 describes the discontinuities present in the capacity cost curve (Point B, and Point C).

NFV provides an economically viable platform for hosting networking applications that help service providers achieve diminished OPEX and CAPEX (Walker, 2012; John, et al., 2017). This research paper identifies the advantages of NFV for service providers and vendors, and proposes a peer production approach for building VNF through a unique interdisciplinary methodology to answer this subproblem. This subproblem identified contractual vulnerabilities present in the vendor proprietary model, as well as mutuality of interests (among service providers and vendors) that is required to commit to a peer production arrangement. Next, the subproblem focused on creating a modular framework for VNF development and identified multiple intrinsic and extrinsic incentives for encouraging employees in peer participation model. Finally, 
through capacity cost curve analysis it was indicated that larger ISPs may gain economic advantage over smaller ISPs by the adoption of containerized VNFs due to the presence of Economies of Scale.

\section{Conclusion and Future Scope}

This research paper answered the research question and subproblems by identifying various contractual vulnerabilities present in the vendor proprietary model. Further, the research analyzed various mutuality of interests (among service providers and vendors) to commit to an open source peer production arrangement and created a modular framework for VNF development. To encourage ISP employees' participation in the peer production participation model, the research identified multiple intrinsic and extrinsic incentives which serve as major contributions of this work. The results from this research also indicated that larger ISPs may gain an economic advantage over smaller ISPs by the adoption of containerized VNFs due to the presence of Economies of Scale found through the capacity cost curve function.

As a part of the future work, this micro-economic research would be extended to analyze the modularity (peer production) regarding the complexity and scope of NFV components to identify the effort required to implement a module using open source. Although the survey results analyzed in this research analyzed peer participation motivations, the future research would extend the survey to include additional responses from a more diverse group of participants which presently lack in the current work.

\section{Conflicts of Interest}

The authors declare no conflicts of interest regarding the publication of this paper.

\section{References}

Ang, S., \& Beath, C. (1993). Hierarchical Elements in Software Contracts. Journal of Organizational Computing, 3, 329-361. https://doi.org/10.1080/10919399309540207

Athey, S., \& Ellison, G. (2014). Dynamics of Open Source Movements. Journal of Economics \& Management Strategy, 23, 294-316. https://doi.org/10.1111/jems.12053

Bauwens, M. (2009). Class and Capital in Peer Production. Capital \& Class, 33, 121-141. https://doi.org/10.1177/030981680909700107

Benkler, Y. (2002). Coase's Penguin, or, Linux and the Nature of the Firm. Yale Law Journal, 112, 369-446. https://www.yalelawjournal.org/article/coases-penguin-or-linux-and-the-nature-of-the -firm https://doi.org/10.2307/1562247

Benkler, Y. (2003). The Wealth of Networks (Ch. 3-4). London: Yale University Press.

Benkler, Y., \& Nissenbaum, H. (2006). Commons-Based Peer Production and Virtue. The Journal of Political Philosophy, 14, 394-419.

https://doi.org/10.1111/j.1467-9760.2006.00235.x 
Besten, M., Dalle, J., \& Gallia, F. (2008). The Allocation of Collaborative Efforts in Open-Source Software. Information Economics and Policy, 20, 316-322. https://doi.org/10.1016/j.infoecopol.2008.06.003

Coase, R. (1937). The Nature of the Firm. Economica, 4, 386-405. http://www3.nccu.edu.tw/ jsfeng/CPEC11.pdf https://doi.org/10.1111/j.1468-0335.1937.tb00002.x

Coase, R. (1959). The Federal Communications Commission. The Journal of Law and Economics, 2, 1-40. https://doi.org/10.1086/466549

Deci, L., \& Koestner, R. (1999). Meta-Analytic Review of Experiments: Examining the Effects of Extrinsic Rewards on Intrinsic Motivation. Psychological Bulletin, 125, 627-668. https://doi.org/10.1037/0033-2909.125.6.627

Dedrick, J., \& West, J. (2003). Why Firms Adopt Open Source Platforms: A Grounded Theory of Innovation and Standards Adoption. In J. L. King, \& K. Lyytinen (Eds.), Proceedings of the Workshop on Standard Making: A Critical Research Frontier for Information Systems (pp. 236-257).

Frey, B. S. (1997). Not Just for the Money: An Economic Theory of Personal Motivation. Cheltenham: Edward Elgar.

Gandotra, R., \& Perigo, L. (2018). SDNMA: A Software-Defined, Dynamic Network Manipulation Application to Enhance BGP Functionality. In 20th IEEE International Conference on High Performance Computing and Communications. IEEE Explore. https://doi.org/10.1109/HPCC/SmartCity/DSS.2018.00167

Gedia, D., \& Perigo, L. (2018a). Performance Evaluation of SDN-VNF in Virtual Machine and Container. In 2018 IEEE Conference on Network Function Virtualization and Software Defined Networks (pp. 1-7). IEEE Explore. https://doi.org/10.1109/NFV-SDN.2018.8725805

Gedia, D., \& Perigo, L. (2018b). A Centralized Network Management Application for Academia and Small Business Networks. Information Technology in Industry, 6, 1-10.

Gedia, D., \& Perigo, L. (2018c). NetO-App: A Network Orchestration Application for Centralized Network Management in Small Business Networks. In 4th International Conference on Computer Science, Engineering and Information Technology (pp. 61-72). IEEE Explore. https://doi.org/10.5121/csit.2018.81106

Hardin, G. (1968). Tragedy of the Commons. Science, 162, 1243-1248. https://doi.org/10.1126/science.162.3859.1243

Hawkins, J. (2017). Automating Service Creation and Provisioning for Creative Ethernet Services. In 2017 Fall Technical Forum (pp. 3-14). Washington: NCTA-The Internet \& Television Association.

https://www.nctatechnicalpapers.com/Paper/2017/2017-automating-service-creation-a nd-provisioning-for-creative-ethernet-services/download

John, H. et al. (2017). Automating Service Creation and Provisioning for Creative Ethernet Services. In Fall Technical Forum. Washington: NCTA-The Internet \& Television Association.

Kogut, B., \& Metiu, A. (2001). Open Source Software Development and Distributed Innovation. Oxford Review of Economic Policy, 17, 248-215.

https://doi.org/10.1093/oxrep/17.2.248

Laghrissi, A., \& Taleb, T. (2018). A Survey on the Placement of Virtual Resources and Virtual Network Functions. IEEE Communications Surveys \& Tutorials, 21, 1409-1434. https://doi.org/10.1109/COMST.2018.2884835 
Lerner, J., \& Tirole, J. (2002). Some Simple Economics of Open Source. The Journal of Industrial Economics, 50, 197-234. https://doi.org/10.1111/1467-6451.00174

Li, Y., \& Chen, M. (2015). Software-Defined Network Function Virtualization: A Survey. IEEE Access, 3, 2542-2553. https://doi.org/10.1109/ACCESS.2015.2499271

Lindenberg, S. (2001). Intrinsic Motivation in a New Light. Kyklos, 54, 317-343. https://doi.org/10.1111/1467-6435.00156

Malone, T., Yates, J., \& Benjamin, R. (1987). Electronic Markets and Electronic Hierarchies. Communications of the ACM, 30, 484-497. https://doi.org/10.1145/214762.214766

Osterloh, M., Rota, S., \& Kuster, B. Open Source Software Production: Climbing on the Shoulders of Giants. Zurich: Institute for Research in Business Administration, University of Zurich.

SDN-NFV World Congress Agenda. https://congress.layer123.com/event/c7ecb486-321f-4812-a554-4cfbd529a159/summary

Valencia, E., Izzo, S., \& Polonsky, B. (2015). How Will NFV/SDN Transform Service Provider Opex? IEEE Network, 29, 60-67. https://doi.org/10.1109/MNET.2015.7113227

Walker, M. (2012). A Growth Opportunity for Vendors: Telco Opex. OVUM. https://www.theedgemarkets.com/article/ovum-slow-growth-ahead-telecom-service-pr ovider-revenues-capex 